\title{
Entry, Descent, and Landing Operations Analysis for the Stardust Re-Entry Capsule
}

\author{
Prasun N. Desai* \\ NASA Langley Research Center, Hampton, VA, 23681-2199 \\ and \\ Dan T. Lyons $\dagger$, Jeff Tooley $\ddagger$, Julie Kangas** \\ Jet Propulsion Laboratory, Pasadena, CA 91109-8099
}

\begin{abstract}
On the morning of January 15, 2006, the Stardust capsule successfully landed at the Utah Test and Training range in northwest Utah returning cometary samples from the comet Wild-2. An overview of the entry, descent, and landing (EDL) trajectory analysis that was performed for targeting during the Stardust Mission Navigation Operations Phase upon final approach to Earth is described. In addition, how the predicted landing location and the resulting overall 99 percentile landing footprint ellipse obtained from a Monte Carlo analysis changed over the final days and hours prior to entry is also presented. The navigation and EDL operations effort accurately delivered the entry capsule to the desired landing site. The final landing location was $8.1 \mathrm{~km}$ from the target, which was well within the allowable landing area.
\end{abstract}

\section{Introduction}

$\mathrm{S}$ tardust, the forth of NASA's Discovery class missions, was launched on February 7, 1999. The spacecraft performed a close flyby of the comet Wild-2 coming within $149 \mathrm{~km}$ of the comet nucleus. The cometary samples were collected by extending a collection tray on a boom into the gas/dust freestream emanating from the comet (Fig. 1), where the particles were trapped in a material called aerogel. Once the collection process was completed, the collection tray was retracted back into the capsule. In addition to collecting cometary particles, Stardust also collected interstellar dust particles during its 7 year journey. Stardust is the first mission to return samples from a comet. Reference 1 gives an overview of the Stardust mission.

Upon Earth return on the morning of January 15, 2006, the entry capsule containing the cometary samples was released from the main spacecraft, and descended through the Earth's atmosphere decelerating with the aid of a parachute for a successful landing at the U.S. Air Force's Utah Test and Training Range (UTTR) in Northwest Utah. Reference 2 describes the Stardust Earth return trajectory strategy.

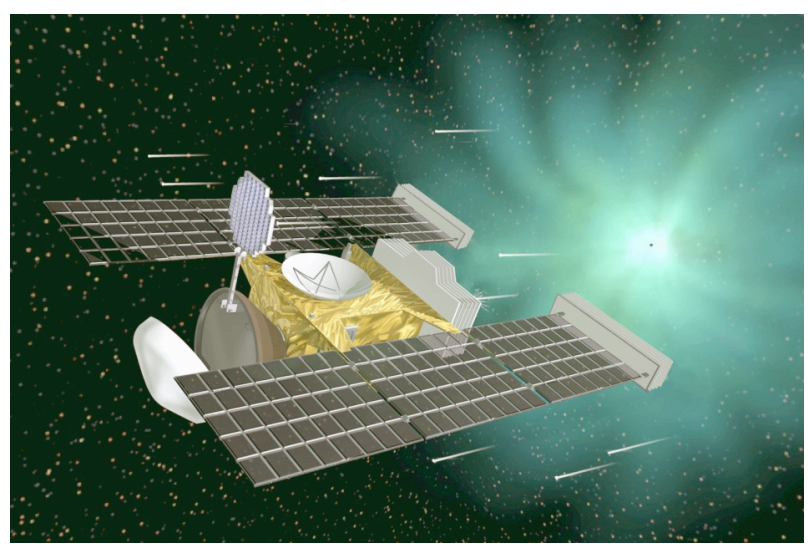

Figure 1. Stardust spacecraft in sample configuration.

\footnotetext{
*Senior Engineer, Exploration Systems Engineering Branch, 1 North Dryden St., MS 489, AIAA Associate Fellow. ${ }^{\dagger}$ Senior Engineer, Entry, Descent, and Landing, Aero Applications Group, 4800 Oak Grove Dr., M/S 301-140L, AIAA Member.

${ }^{\star}$ Engineer, Entry, Descent, and Landing, Aero Applications Group, 4800 Oak Grove Dr., M/S 301-220G, nonmember of AIAA.

${ }^{* *}$ Engineer, Entry, Descent, and Landing, Aero Applications Group, 4800 Oak Grove Dr., M/S 301-150, nonmember of AIAA.
} 
This paper provides an overview of the entry, descent, and landing (EDL) trajectory analysis that was performed for targeting the capsule to UTTR during the Stardust Mission Navigation Operations Phase upon final approach to Earth. In addition, how the predicted landing location and the resulting overall 99 percentile footprint ellipse (obtained from a Monte Carlo analysis) changed over the final days and hours prior to entry is also presented. This analysis was required in order to substantiate the robustness of the capsule descent to assure that all entry mission and public safety requirements were satisfied prior to gaining authorization for capsule separation from the main spacecraft for Earth entry.

\section{Capsule Overview}

The Stardust capsule (Fig. 2) is approximately $0.8 \mathrm{~m}$ in diameter. Its forebody is a blunted $60 \mathrm{deg}$ half-angle sphere-cone. The afterbody is a $30 \mathrm{deg}$ truncated cone. The entry velocity for the Stardust capsule was the highest (inertial velocity of $12.9 \mathrm{~km} / \mathrm{s}$ ) of any Earth returning mission to date. For comparison, the Apollo lunar missions had entry velocities of $11.0 \mathrm{~km} / \mathrm{s}$. This high entry velocity resulted in the highest heating rates for any Earth returning vehicle. Traditional carbon-phenolic based thermal protection systems (TPS) are very effective at such intense heating levels; however, they are quite heavy. To remain within project mass limits, a new lightweight heatshield material Phenolic Impregnated Carbon Ablator (PICA) was utilized. ${ }^{3}$ Reference 4 provides an overview of the Stardust capsule and its design.

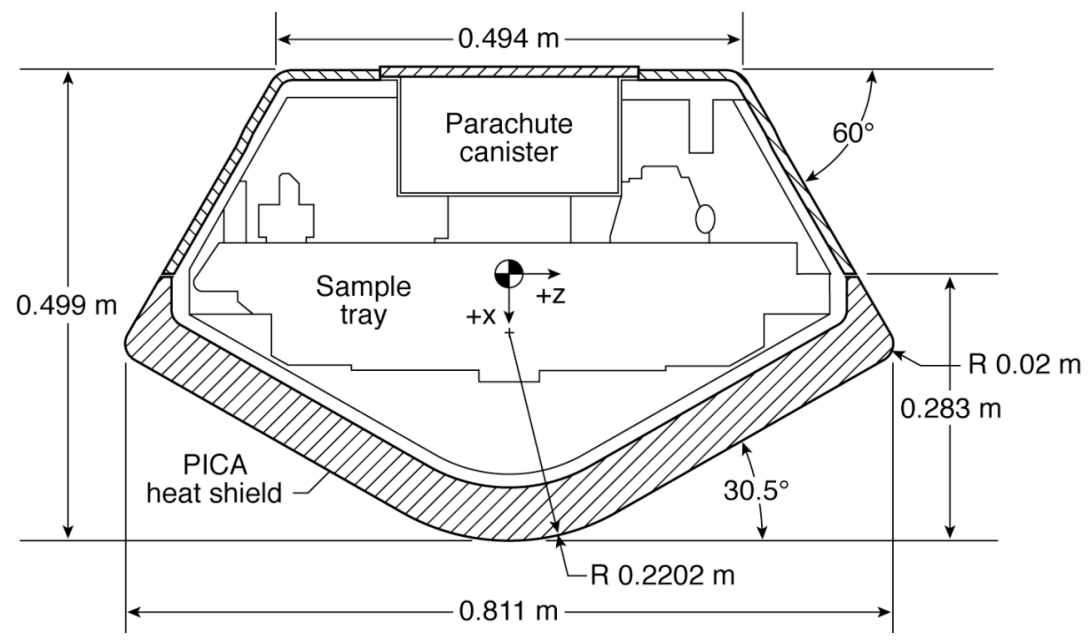

Figure 2. Stardust capsule configuration.

\section{EDL Overview}

Figure 3 shows the nominal Stardust entry sequence, with the terminal descent phases highlighted. Four hours prior to entry, the $45.8 \mathrm{~kg}$ capsule was spun-up to $13.5 \mathrm{rpm}$ and separated from the main spacecraft. The capsule has no active guidance or control systems, so the spin-up is required to maintain its entry attitude ( 0 deg angle-of-attack) during coast. Throughout the atmospheric entry, the passive capsule relies solely on aerodynamic stability for performing a controlled descent through all aerodynamic flight regimes: free molecular, hypersonic-transitional, hypersonic-continuum, supersonic, transonic, and subsonic. The capsule must possess sufficient aerodynamic stability to overcome the gyroscopic (spin) stiffness in order to minimize any angle-of-attack excursions during the severe heating environment. Additionally, this stability must persist through the transonic and subsonic regimes to maintain a controlled attitude at drogue and main parachute deployment. The inertial entry velocity and flight-path angle for Stardust were $12.9 \mathrm{~km} / \mathrm{s}$ and $-8.2 \mathrm{deg}$, respectively. 


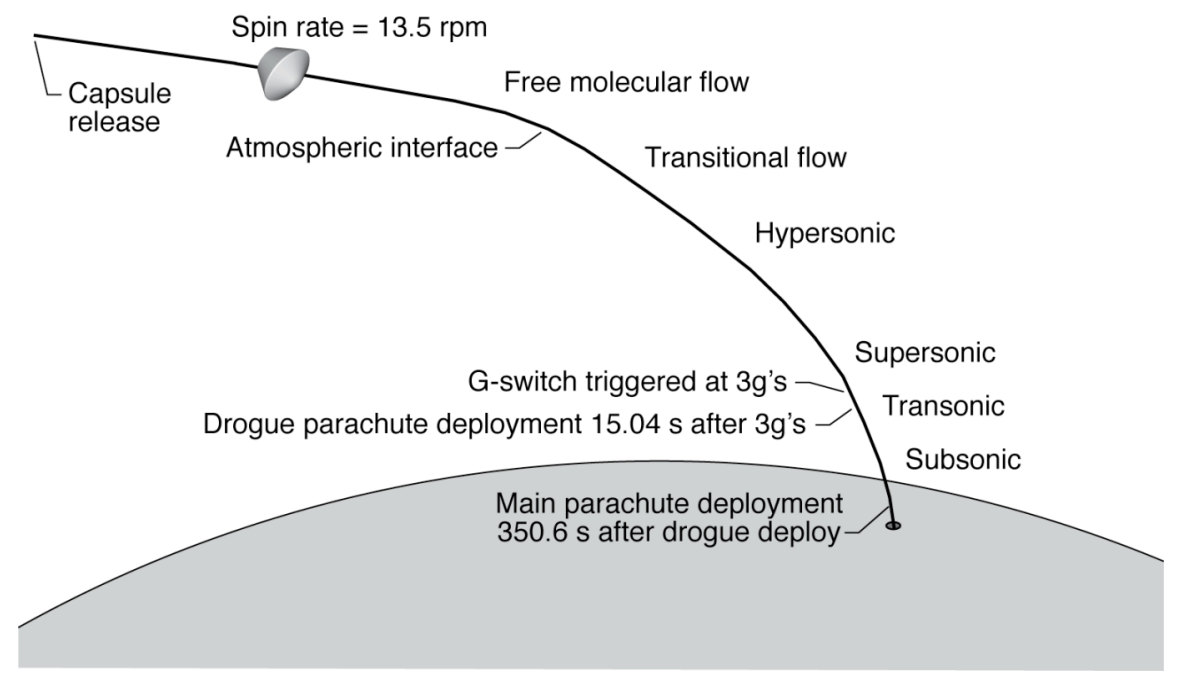

Figure 3. Nominal Stardust capsule entry sequence.

Reference 5 provides an in-depth description on the development of the entry sequence; specifically, the use of the high spin rate and a supersonic drogue parachute. During descent, the capsule utilizes a g-switch (i.e., gravityswitch) and two timers for deployment of the drogue and main parachutes. The g-switch is triggered after sensing 3 g's (on the decelerating side). At that point, the drogue timer is initiated. After 15.04 seconds, the drogue parachute is deployed (approximately Mach 1.37), and the main timer is initiated. After 350.6 seconds (approximately at an altitude of $3.1 \mathrm{~km}$ mean sea level [1.8 km above ground]), the main parachute is deployed to slow the capsule for landing at UTTR. This nominal entry sequence is sufficiently robust to accommodate off-nominal conditions during the descent as shown by the Monte Carlo dispersion analyses in Ref. 5.

\section{Earth Return Strategy}

The Stardust event timeline for final Earth approach is shown in Figure 4, which highlights the Trajectory Correction Maneuvers (TCM) that were baselined for attaining the proper entry conditions. Reference 2 provides an overview of the Earth return strategy showing all of the required TCMs. Prior to TCM-18, which occurred at entry (E) minus 10 days (d), the Stardust return trajectory was on a path that missed the Earth. Only after TCM-18 was successfully executed did the trajectory of the spacecraft become targeted to the Earth (within the atmosphere).

Final targeting was accomplished with TCM-19 at E-36 hours (hr), which placed the nominal landing location in the eastern portion of UTTR. If TCM-19 had not executed or only partially executed, a contingency maneuver TCM-19a or TCM-19b would have been implemented at E-12 hours to achieve the desired target landing location. At E-4 hours, the capsule was separated from the main spacecraft, thus starting the EDL sequence illustrated in Fig. 3 The separation maneuver imparted to the capsule the remaining delta-v required to target the desired nominal landing location at the center of UTTR. At E-3.7 hours, a TCM was performed to divert the main spacecraft into an orbit ahead of the Earth. If TCM-18, 19, or 19a or 19b had all been unsuccessful, the capsule/main spacecraft would have flown by the Earth as depicted in Fig. 4 by the solid line. During Mission Operations, both TCM-18 and TCM19 executed very successfully, as did the separation and divert maneuvers. As a result, the desired entry conditions were achieved with amazing accuracy.,6,7 


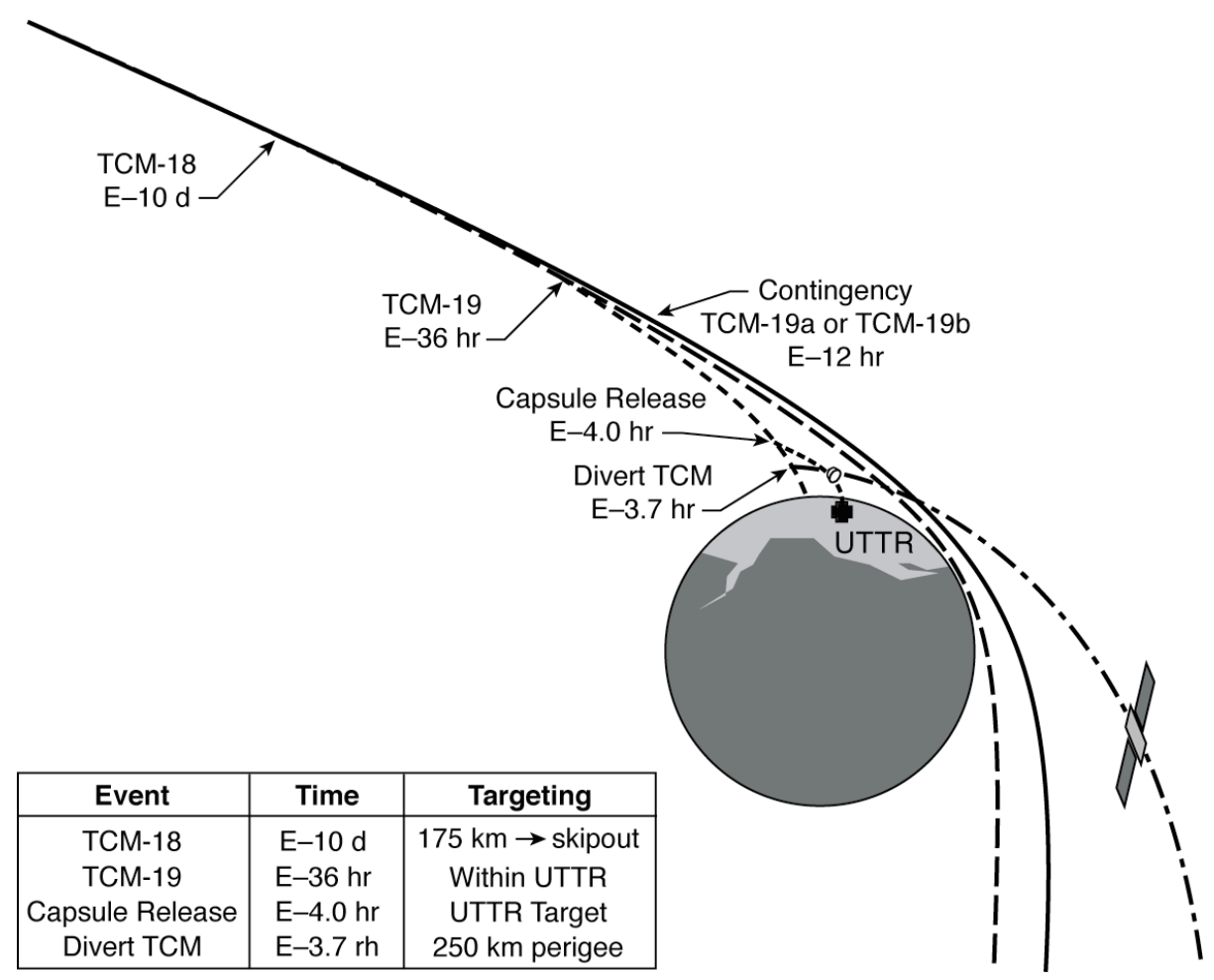

Figure 4. Stardust final Earth approach event timeline.

\section{Trajectory Simulation}

\section{A. Entry Trajectory Requirements and Constraints}

The Stardust atmospheric entry trajectory was designed to fit within an envelope of derived requirements and physical constraints based upon the capsule hardware design. As such, for a successful landing, all entry requirements must be satisfied. Table 1 lists all the EDL requirements and their specific bounds. Monte Carlo dispersion analyses, described in subsequent sections, were performed during the Mission Operations Phase to assess the satisfaction of these requirements.

Table 1: EDL Requirements and Constraints

\begin{tabular}{lc}
\hline \multicolumn{1}{c}{ Requirement } & Limit \\
\hline Entry Flight-Path Angle Error, deg & $< \pm 0.08$ \\
Entry Attitude, deg & $<10$ \\
Max Heat Rate, W/cm & $<1200$ \\
Attitude at Max Heat Rate, deg & $<10$ \\
Max Heat Load, KJ/cm ${ }^{2}$ & $<32.0$ \\
Max Deceleration, Earth g & $<40$ \\
Drogue Chute Deployment Attitude, deg & $<30$ \\
Drogue Chute Deployment Mach Number & $>1.2 \&<1.6$ \\
Landed Footprint, km & $<84$ \\
\hline
\end{tabular}




\section{B. Monte Carlo Uncertainty Sources}

During the entry, off-nominal conditions may arise that affect the descent profile. These off-nominal conditions can originate from numerous sources: state vector uncertainties, capsule mass property measurement uncertainties; separation attitude and attitude rate uncertainties; limited knowledge of the entry day atmospheric properties (density and winds); uncertainty with the aerodynamics; and uncertainties with parachute deployment. In the analysis, an attempt was made to conservatively quantify and model the degree of uncertainty in each mission parameter. For this entry, 41 potential uncertainties were identified. ${ }^{5}$ Table 2 captures these uncertainty sources, along with their corresponding 3- $\sigma$ variances. The subsequent subsections describe in greater detail a few of the uncertainty sources.

Table 2: Monte Carlo Analysis Variables

\begin{tabular}{|c|c|c|}
\hline Variable & 3- $\sigma$ Variation & Distribution \\
\hline Entry states & Based on covariance (See Ref. 6) & - \\
\hline Radial center-of-mass offset, $\mathrm{mm}$ & \pm 0.254 & Gaussian \\
\hline Axial center-of-mass, mm & \pm 0.254 & Gaussian \\
\hline Moments of Inertia (Ixx, Iyy, Izz) & $\pm 2 \%, \pm 5 \%, \pm 5 \%$ & Gaussian \\
\hline Cross products (Ixy, Ixz, Iyz), kg-m² & $\pm 0.003, \pm 0.003, \pm 0.003$ & Gaussian \\
\hline Separation pitch and yaw attitude, deg & $\pm 0.56, \pm 0.56$ & Gaussian \\
\hline Separation pitch and yaw rates, $\mathrm{deg} / \mathrm{s}$ & $\pm 2.83, \pm 2.83$ & Uniform \\
\hline Separation roll rate, $\mathrm{deg} / \mathrm{s}$ & \pm 12.0 & Gaussian \\
\hline Aerodynamic coefficients & See Ref. 5 & - \\
\hline Ablation mass loss & $\pm 10 \%$ & Gaussian \\
\hline Drogue Parachute Drag Coefficient & $\pm 10 \%$ & Uniform \\
\hline Main Parachute Drag Coefficient & $\pm 10 \%$ & Uniform \\
\hline G-switch acceleration trigger value & $\pm 10 \%$ & Uniform \\
\hline Drogue parachute timer, $\mathrm{s}$ & \pm 0.05 & Uniform \\
\hline Main parachute timer, s & \pm 0.05 & Uniform \\
\hline Atmosphere & GRAM-95 model (See Ref. 8) & - \\
\hline
\end{tabular}

\section{Entry Covariance}

The Stardust strategy for Earth approach was designed to maximize public safety in light of possible anomalies and contingencies, while still preserving the capability to meet the entry requirements. As a result, a series of maneuvers were performed to set up the approach and entry (see Ref. 2). Initial conditions at entry were obtained from orbit determination solutions performed by the Stardust Navigation Team. References 2, 6, and 7 provide a description of the navigation process during the return phase, and the determination of the final arrival conditions prior to entry. The navigation accuracy obtained for Stardust yielded extremely small state errors upon Earth arrival. The final orbit determination solution produced an inertial entry flight-path angle of $-8.21 \mathrm{deg}$ with a 3- $\sigma$ error of $\pm 0.0017 \mathrm{deg}$, which was well within the $\pm 0.08 \mathrm{deg}$ requirement.

\section{Capsule/Cruise-Stage Separation}

Based on the final main spacecraft and capsule mass properties, a statistical separation analysis was performed to predict separation attitude and attitude rate errors. The attitude errors predicted in pitch and yaw were $\pm 0.56 \mathrm{deg}$ in each axes. The attitude pitch and yaw rate errors were $\pm 2.83 \mathrm{deg} / \mathrm{s}$ in each axes, and the roll rate error was \pm 12.0 $\mathrm{deg} / \mathrm{s}$. These variations were used as inputs in the Monte Carlo analysis.

3. Atmosphere Model

The Earth atmosphere model utilized by Stardust for the entry trajectory design and analysis was the Global Reference Atmospheric Model-1995 Version (GRAM-95). ${ }^{8}$ This model is an amalgam of three empirically based global data sets of the Earth that can produce an atmosphere profile as a function of altitude for a given date, time, and positional location about the Earth. GRAM-95 produces a representative atmosphere taking into account variations in diurnal, seasonal, and positional information for a given trajectory to produce nominal density, temperature, and pressure profiles and their statistical perturbations along the trajectory flight track. GRAM-95 is not a predictive model. A profile is generated based on historical data for a given time, season, and location. 
Figure 5 shows samples of few randomly perturbed density profiles as a percentage of the nominal profile for the Stardust entry date of January 15, 2006 produced by the GRAM-95 model. Also, depicted are the upper and lower $( \pm 3-\sigma)$ boundaries of the possible density variation. As seen, density variations between $\pm 5 \%$ to $\pm 25 \%$ are possible. In addition, GRAM-95 can also produce nominal wind profiles and their statistical perturbations for the Northward, Eastward, and vertical wind components. Figures 6 and 7 show the nominal as well as a few randomly sampled wind profiles (for the Stardust entry date of January 15, 2006) for the Northward and Eastward wind components, respectively, along with their upper and lower $( \pm 3-\sigma)$ boundaries. Also depicted is the actual wind profile obtained from balloon measurements 2 hours prior to entry (E-2 hr), which will be discussed in a subsequent section. In the Monte Carlo analysis, an atmosphere profile (density and wind components) was randomly generated for each case having the characteristics shown in Figs. 5-7.

\section{Trajectory Analysis}

Two trajectory propagation codes were utilized for the Stardust landing dispersion analyses: the Program to Optimize Simulated Trajectories (POST) program ${ }^{9}$ and the Atmospheric-Entry Powered Landing (AEPL) program. ${ }^{10}$ Two codes were employed to obtain independent verification that the predicted nominal landing location and the overall size of the dispersed landing footprint ellipse were within the UTTR boundaries to ensure public safety.

The POST trajectory analysis was performed modeling six-degree-of-freedom (6DOF) dynamics, which included all forces and torques on the spacecraft, from atmospheric interface to drogue parachute deployment. During this portion of the entry, the full set of capsule aerodynamics and mass properties were incorporated into the simulation to accurately model the hypersonic descent. ${ }^{5}$ From drogue parachute deployment to landing, three-degree-of-freedom (3DOF) dynamics were used, in which only the drag force was modeled and was assumed to act opposite the wind-relative velocity vector. The POST trajectory simulation seamlessly transitions from $6 \mathrm{DOF}$ to $3 \mathrm{DOF}$ dynamics within a single continuous simulation.

The version of the AEPL program used for Stardust employed 3DOF analyses throughout. Since the Stardust entry was unguided and uncontrolled, the 3DOF results from AEPL agreed well with the POST 6DOF/3DOF simulation. The POST results were baselined as prime for the mission. In general, there was very good agreement between the two simulations.

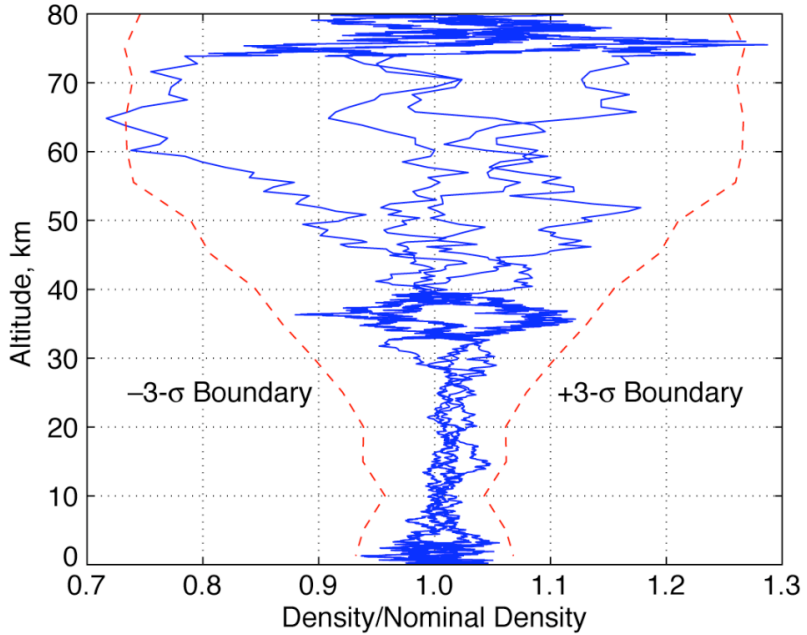

Figure 5. Density variation from GRAM-95 model.

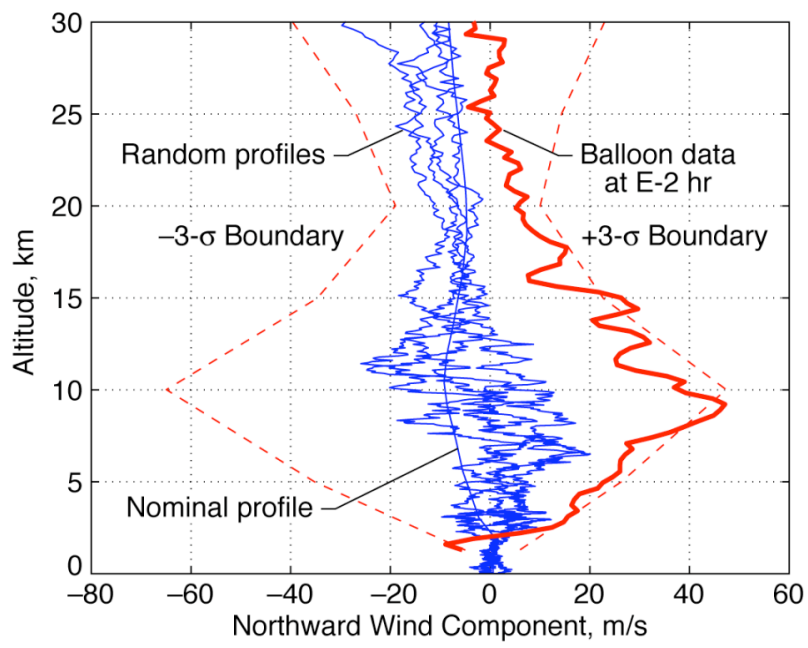

Figure 6. Northward wind component variation from GRAM-95 model.

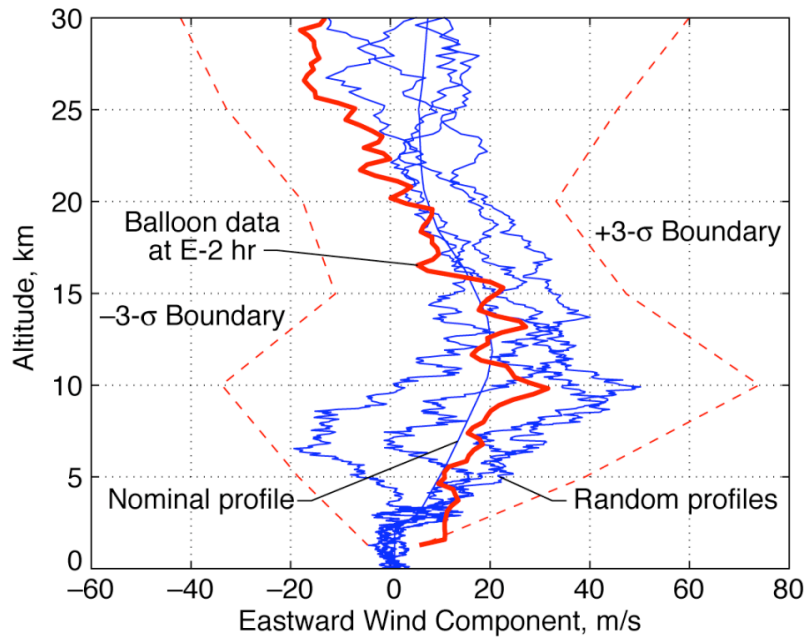

Figure 7. Eastward wind component variation from GRAM-95 model. 


\section{Monte Carlo Dispersion Analysis}

A Monte Carlo dispersion analysis was utilized to statistically assess the robustness of the Stardust entry to offnominal conditions to assure that all EDL requirements and constraints were satisfied (see Table 1). All the input variables listed in Table 2 were randomly varied in the Monte Carlo dispersion analysis within their respective variances and distribution types. The analysis included uncertainties in the initial state vector, capsule mass properties (mass, center-of-gravity, inertia), separation attitude and attitude rates, aerodynamic coefficients, ablation mass loss, atmospheric density and winds, parachute drag, g-switch trigger value, and parachute deployment timers.

For the dispersion analysis, 3000 random off-nominal cases were run for all the navigation orbit determination (OD) solutions that were computed ${ }^{2,6}$ at the various event times during the Mission Operations Phase. This analysis was performed to determine the appropriate magnitude and direction for the TCM-18 and TCM-19 maneuvers for proper targeting to UTTR. In addition, this analysis was used to assess the OD solution stability and to understand the movement of the nominal landing location and the variation in the 99 percentile footprint ellipse size within UTTR. This understanding was crucial in order to gain authorization for capsule separation and the subsequent Earth entry. The size of the 99 percentile footprint ellipse obtained from the Monte Carlo dispersion analysis was used in a public safety probabilistic analysis to certify that the risks of the Stardust capsule entry were acceptable. Reference 11 describes the hazard analysis that was performed for the Stardust entry capsule using the 99 percentile footprint ellipses generated by this Monte Carlo analysis. This hazard analysis was preformed for the nominal scenario of an intact capsule, as well as for a burn-up and breakup scenario in case of capsule failure during the entry.

The Monte Carlo dispersion analysis was performed on all the post-TCM18 OD solutions (OD s06008a through OD s06014a). Figure 8 shows the corresponding results at landing assuming that a perfect TCM-19 is executed. For clarity, only the results for three OD solutions are shown (OD s06011d, OD s06012b2, and OD s06014a), where their nominal landing locations (center points) and the 99 percentile footprint ellipses at UTTR are depicted. The target location selected for Stardust is near the center of UTTR having the coordinates 246.55 deg East Longitude and 40.3167 deg North Latitude.

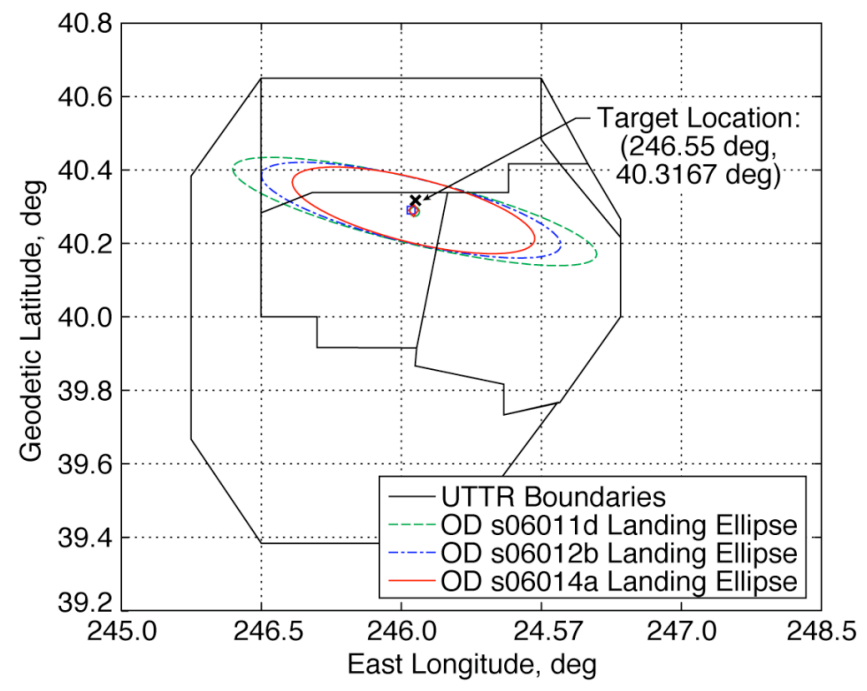

Figure 8. Landing locations for post-TCM-18 OD solutions.

Over the course of the post-TCM-18 OD solutions, the nominal predicted landing location is observed to be stable with little drift. The nominal predicted landing locations are hard to differentiate, as they lie nearly on top of each other. The benefit of stable OD solutions is that greater confidence can be placed on the footprint size refinement. For the post-TCM-18 OD solutions, the 99 percentile footprint ellipses decrease in size. The footprint size decreases from $113.3 \mathrm{~km}$ in downrange for OD s06011d to $75.5 \mathrm{~km}$ for OD s06014a. Table 3 summaries the variation in the 99 percentile landed footprint ellipse sizes. The corresponding inertial flight-path angle errors at atmospheric interface for these three OD solutions show a similar trend and are also summarized in Table 3. 
Table 3: Variation in Landing Footprint Ellipse Size

\begin{tabular}{ccc}
\hline OD Solution & $\begin{array}{c}\text { 99 Percentile } \\
\text { Footprint Size }(\mathbf{k m})\end{array}$ & $\begin{array}{c}\text { 3- } \boldsymbol{\sigma} \text { Flight-Path } \\
\text { Angle Error (deg) }\end{array}$ \\
\hline Post-TCM-18 & $113.3 \times 19.7$ & \\
s06011d & $93.2 \times 19.5$ & \pm 0.144 \\
s06012b2 & $75.5 \times 19.4$ & \pm 0.115 \\
s06014a & & \pm 0.083 \\
Post-TCM-19 & $52.7 \times 19.3$ & \\
s06014c & $47.5 \times 19.2$ & \pm 0.033 \\
s06015a & $45.1 \times 19.2$ & \pm 0.021 \\
s06015f & $38.1 \times 8.2$ & \pm 0.0017 \\
\hline \hline
\end{tabular}

Similarly, Monte Carlo analyses were performed for all the post-TCM-19 OD solutions (OD s06014b through OD s06014f). Figure 9 depicts the landing locations for a few of these OD solutions (OD s06014c, OD s06015a, and OD s06015f). All the OD solutions post-TCM-19 were extremely stable and produced nearly identical landing locations as observed in Fig. 9, where the 99 percentile landing ellipses lie nearly on top of each other. The results for OD s06015f (which was the last OD solution available prior to entry) showed that all the EDL requirements and constraints were well within their limits, and that the final predicted nominal landing location was very close to the desired target (only $5.7 \mathrm{~km}$ away). The footprint size for these OD solutions decreases from $52.7 \mathrm{~km}$ in downrange for OD s06014c to $45.1 \mathrm{~km}$ in downrange for OD s06015f. Table 3 summaries the landed footprint variation, along with the variation in the corresponding inertial flight-path angle error. As seen, the inertial flight-path angle for OD $\mathrm{s} 06015 \mathrm{f}$ was extremely small having a value of $\pm 0.0017 \mathrm{deg}$. As such, the Navigation Team accurately delivered the capsule to the desired entry conditions. Based on OD s06015f results of the predicted nominal landing location being $5.7 \mathrm{~km}$ away from the desired target and with a 99 percentile footprint ellipse of $45.1 \mathrm{~km}$ by $19.2 \mathrm{~km}$, the authorization for capsule separation and subsequent Earth entry was granted.

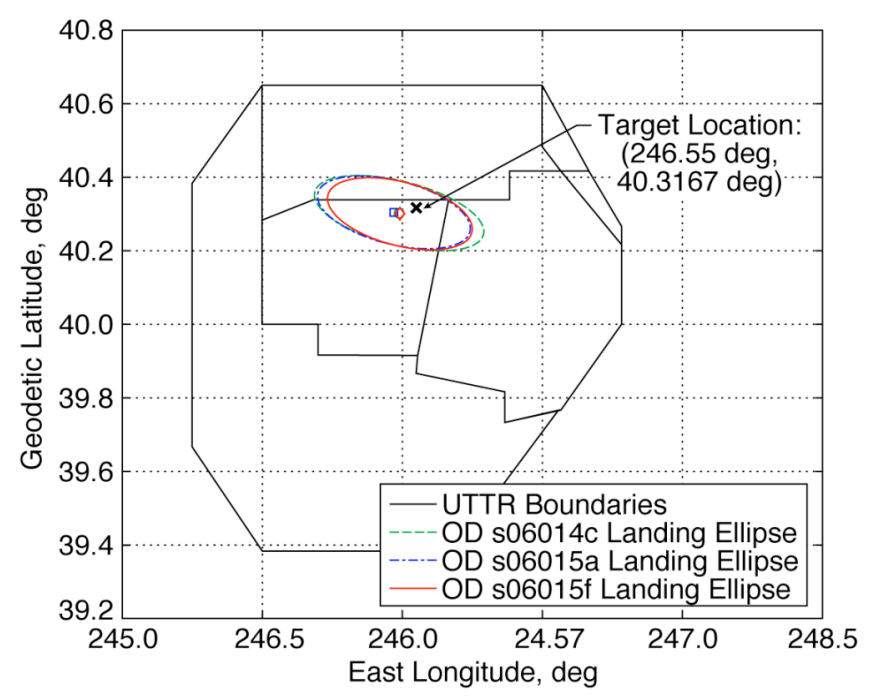

Figure 9. Landing locations for post-TCM-19 OD solutions.

Unfortunately, on entry day of January 15,2006 , a winter storm was moving through western Utah, which was producing very strong winds over UTTR. The effect of strong winds would cause the capsule during parachute descent to drift from its predicted landing location. To address such a scenario, the Stardust Entry Operations strategy had baselined two balloon launches (at E-8 hours and E-2 hours) to obtain measurements of the actual winds that would be observed during the entry in an effort to better predict the landing location and footprint ellipse. These two balloon measurement data revealed that very strong sustained winds were present over UTTR due to the winter bliz- 
zard. Figures 6 and 7 show the actual wind profiles measured at E-2 hours. Note, although the balloon measurement data had a timestamp of E-2 hours, the actual measurements were taken approximately at E-4 hours due to the logistics and processing time requirements. As seen in Fig. 6, a very strong sustained wind to the North was present at E2 hours. This sustained Northward wind component had a peak value of $50 \mathrm{~m} / \mathrm{s}$, and corresponded to approximately a 3- $\sigma$ high profile from the GRAM-95 model. Although, the storm winds were predicted to subside over the remaining two hours prior to landing, this sustained Northward wind would tend to push the capsule landing location towards the North. Consequently, an updated prediction of the nominal landing location (using this balloon measured wind data) was necessary to aid the retrieval of the capsule by notifying the recovery team of the change in nominal landing location.

Using this E-2 hour balloon measurement wind data, the Monte Carlo dispersion analysis was repeated with the GRAM-95 wind dispersions being replaced with the E-2 hour wind profile. Figure 10 shows the updated predicted nominal landing location and 99 percentile footprint ellipse. As seen, the OD s06015f landing prediction shifted North due to the E-2 hour balloon measurement wind data. The predicted nominal landing location moved from 5.7 $\mathrm{km}$ West-Southwest of the target (using the GRAM-95 wind profiles) to $9.4 \mathrm{~km}$ North-Northwest of the target. The updated 99 percentile footprint ellipse was $38.1 \mathrm{~km}$ by $8.2 \mathrm{~km}$. The Northward wind shifted the predicted nominal landing location approximately $11.5 \mathrm{~km}$ due North. The actual final landing location is shown in Fig. 10 and was 8.1 $\mathrm{km}$ North-Northwest of the desired target, and was within the OD s06015f pre-entry predicted 99 percentile landing ellipse. Although, the actual final landing location indicated that the capsule had not drifted as much to the North as the updated prediction, this outcome was undoubtedly due to the winds subsiding (as forecasted) from that balloon measured data at E-2 hour, which was used in the update prediction.

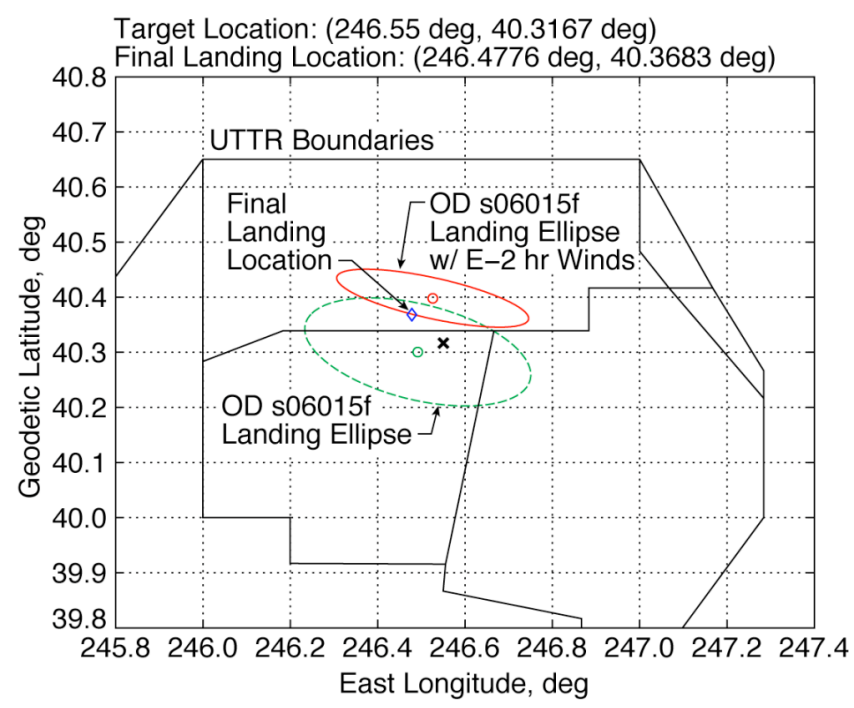

Figure 10. Final capsule landing location.

\section{Conclusion}

On the morning of January 15, 2006, the Stardust entry capsule successfully descended through the Earth's atmosphere decelerating with the aid of a parachute and landed at the Utah Test and Training Range completing a 7 year journey that returned cometary samples from the comet Wild-2. The navigation and the entry, descent, and landing trajectory analyses that were performed during the Mission Operations Phase upon final approach to Earth accurately delivered the entry capsule to the desired landing site. The capsule landed $8.1 \mathrm{~km}$ from the desired target, and was well within the allowable landing area at the Utah Test and Training Range. As a result, the process of targeting a capsule from deep space and accurately landing it on Earth was successfully demonstrated. Preliminary indications are that the actual entry trajectory was very close to the pre-entry prediction. As a result, the design principles and methodologies utilized for the entry flight dynamics, aerodynamics, and aerothermodynamics analyses were corroborated. 


\section{Acknowledgments}

A portion of this research was carried out at the Jet Propulsion Laboratory, California Institute of Technology, under a contract with the National Aeronautics and Space Administration. Reference herein to any specific commercial product, process, or service by trade name, trademark, manufacturer, or otherwise, does not constitute or imply its endorsement by the United States Government or the Jet Propulsion Laboratory, California Institute of Technology.

\section{References}

${ }^{1}$ Atkins, K. L., Brownlee, D. E., Duxbury, T., Yen, C. W., Tsou, P., and Vollinga, J. M., "STARDUST: Discovert's InterSteller Dust and Cometary Sample Return Mission," 1997 IEEE Aerospace Conference, Proceeding Vol. 4 (A97-44051 12-99), Apsen, CO, February1997, pp. 229-245.

${ }^{2}$ Helfrich, C., Bhat, R., Kangas, J., Wilson, R., Wong, M., Potts, C., and Williams, K., "Maneuver Analysis and Targeting Strategy for the Stardust Re-Entry Capsule," AIAA/AAS Astrodynamics Specialists Conference, AIAA, Washington D.C., Aug. 2006 (to be published).

${ }^{3}$ Tran, H., Johnson, C., Rasky, D., Hui, F., Chen, Y.K., and Hsu, M., "Phenolic Impregnated Carbon Ablators (PICA) for Discovery Class Missions,” AIAA Paper No. 96-1911, June, 1996.

${ }^{4}$ Willcockson, W., "Stardust Sample Return Capsule Design Experience," Journal of Spacecraft and Rockets, Vol. 36, No. 3, May-June 1999, pp. 470-474.

${ }^{5}$ Desai, P. N., Mitcheltree, R. A., and Cheatwood, F.,M., "Entry Dispersion Analysis of the Stardust Sample Return Capsule," Journal of Spacecraft and Rockets, Vol.36, No. 3, May-June 1999, pp. 463-469.

${ }^{6}$ Baird, D., Bhaaskaran, S., Jah, Moriba., Jeffereson, D., Kennedy, B., Lewis, G., Martin-Mur, T., McElrath, T., Mottinger, N. Nandi, S., and Thompson, P., "Stardust Earth Return Orbit Determination," AIAA/AAS Astrodynamics Specialists Conference, AIAA, Washington D.C., Aug. 2006 (to be published).

${ }^{7}$ Kennedy, B., Nandi, M., and McElrath, T. "Modeling of Deadband Delta-V for the Stardust Earth Return: Calibration, Analysis, Prediction, and Performance," AIAA/AAS Astrodynamics Specialists Conference, AIAA, Washington D.C., Aug. 2006 (to be published).

${ }^{8}$ Justus, C. G., Jeffries III, W. R., Yung, S. P., and Johnson, D. L., "The NASA/MSFC Global Reference Atmospheric Model 1995 Version (GRAM-95)," NASA TM-4715, Aug. 1995.

${ }^{9}$ Brauer, G. L., Cornick, D. E., and Stevenson, R., "Capabilities and Applications of the Program to Optimize Simulated Trajectories (POST)," NASA CR-2770, Feb. 1977.

${ }^{10}$ Klumpp, A. R., Atmospheric-Entry, Powered Landing Simulator V4.10C User's Guide, April 23, 2003 (JPL Internal Document).

${ }^{11}$ Tooley, J., Desai, P. N., Lyons, D. T., Hirst, E., Wahl, T., Ivano, M., and Wawrzyniak, G., "Landing and Population Hazard Analysis for Stardust Entry in Operations and Entry Planning," AIAA/AAS Astrodynamics Specialists Conference, AIAA, Washington D.C., Aug. 2006 (to be published). 